\title{
recillunds
}

Revista Científica Mundo de la Investigación y el Conocimiento

Miguel Ángel Pozo Arcentales a; María José Cedeño Zambrano ${ }^{\text {b; }}$ Karol Natalie Galarza Avila ${ }^{c}$; Luis Javier Alvarez Moyon ${ }^{\mathrm{d}}$

Fibrilación auricular: factores de riesgo, diagnóstico y tratamiento

Atrial fibrillation: risk factors, diagnosis and treatment

Revista Científica Mundo de la Investigación y el Conocimiento. Vol. 3 núm.3. Esp., noviembre, ISSN: 2588-073X, 2019, pp. 529-547

DOI: $10.26820 /$ recimundo/3.(3.Esp).noviembre.2019.529-547

URL: http://recimundo.com/index.php/es/article/view/622

Código UNESCO: 3205 Medicina Interna

Tipo de Investigación: Artículo de Revisión

(C) RECIMUNDO; Editorial Saberes del Conocimiento, 2019

Recibido: 15/09/2019

Aceptado: 23/10/2019

Publicado: 30/11/2019

Correspondencia: majocezam@ @otmail.com

a. Médico; Investigador Independiente; Guayaquil, Ecuador.

b. Licenciado en Psicología; Investigadora Independiente; Guayaquil, Ecuador; majocezam@ hotmail.com

c. Médico; Investigadora Independiente; Guayaquil, Ecuador; karito-naty@ hotmail.com

d. Médico; Investigador Independiente; Guayaquil, Ecuador; lujam898989@gmail.com 


\section{Fibrilación auricular: factores de riesgo, diagnóstico y tratamiento}

Vol. 3, núm. 3 Esp., (2019)

Miguel Ángel Pozo Arcentales; María José Cedeño Zambrano; Karol Natalie Galarza Avila; Luis Javier Alvarez Moyon

\section{RESUMEN}

La fibrilación auricular es la arritmia más frecuente. Su clasificación según el patrón y tipo clínico permiten decidir el tratamiento a emplear que debe incluir control de síntomas y prevención de cardioembolismo. La elección del tratamiento depende de la presencia de fenómenos desencadenantes de la arritmia, factores de riesgo para tromboembolismo, factores de riesgo para sangrado, función cardiaca, funcionalidad del paciente, costoefectividad de los medicamentos y facilidades para acceder al sistema de salud. El anticoagulante a usar deberá elegirse con base en el tipo de fibrilación auricular y la presencia de contraindicaciones, documentación de anticoagulación inefectiva o alto riesgo de falla al tratamiento con warfarina. En presencia de contraindicaciones para la anticoagulación esta puede emplearse en pacientes con alto riesgo de sangrado cuando los factores de riesgo son controlables o corregibles, pudiéndose ofrecer como alternativa la oclusión de la orejuela auricular en pacientes que persisten con alto riesgo de sangrado.

Palabras claves: Anticoagulación; Arritmias Cardíacas; Fibrilación Auricular. 
Fibrilación auricular: factores de riesgo, diagnóstico y tratamiento

Vol. 3, núm. 3 Esp., (2019)

Miguel Ángel Pozo Arcentales; María José Cedeño Zambrano; Karol Natalie Galarza Avila; Luis

Javier Alvarez Moyon

\begin{abstract}
Atrial fibrillation is the most frequent arrhythmia. Its classification according to pattern and clinical type allows to decide the therapeutic strategy to use, that most include control of symptoms and prevention of cardioembolic events. The election of the treatment depends on the presence of triggering events, risk factors for thromboembolism, risk factors for bleeding, cardiac function, patient funcionality, medication costoefectiveness and health care access. The type of anticoagulant has to be supported on the type of atrial fibrillation and the presence of contraindications, documented ineffective anticoagulation or high risk of failure to warfarin. In case of contraindications for anticoagulation this could still be used in high bleeding risk patients, when risk factors are controllable or corrected; leaving left atrial appendage closure as an option for patients that remain in high risk for bleeding events.
\end{abstract}

Key words: Anticoagulation; Atrial fibrillation; Arrhythmias, Cardiac. 


\section{Fibrilación auricular: factores de riesgo, diagnóstico y tratamiento}

Vol. 3, núm. 3 Esp., (2019)

Miguel Ángel Pozo Arcentales; María José Cedeño Zambrano; Karol Natalie Galarza Avila; Luis Javier Alvarez Moyon

\section{Introducción.}

La fibrilación auricular (FA) es considerada un problema de salud pública. Su prevalencia se multiplica con la edad y es un factor de riesgo directo para enfermedad cerebrovascular (ECV), insuficiencia cardiaca, infarto agudo de miocardio (IAM) y muerte (Andrade, y otros, 2016).

En Colombia, estudios recientes evidencian aumento en su incidencia y mortalidad, afectando principalmente a personas de sexo masculino de la séptima década de la vida (Roselli, Rodríguez, García, \& Rueda, 2013). El aumento de la esperanza de vida y la enfermedad cardiovascular como la primera causa de muerte, hace esperable que cualquier medico pueda entrar en contacto con estos pacientes; algunos de ellos con secuelas definitivas secundarias a cardioembolismos prevenibles.

Las últimas guías de práctica clínica en fibrilación auricular de diferentes latitudes enfatizan la importan-cia de un diagnóstico oportuno y manejo mediante un programa interdisciplinario centrado en el paciente (Pava-Molano \& Perafán-Bautista, 2016).

Este artículo entrega un resumen de la mejor evidencia disponible sobre fibrilación auricular, entregando de forma comprensible a todo el personal de salud la información más relevante a tener en cuenta a la hora de atender pacientes con este diagnóstico, asistiendo la toma de decisiones durante su práctica clínica.

La FA es la arritmia supraventricular más frecuente en el ser humano y se caracteriza por la pérdida de la actividad auricular eléctrica mecánica organizada. Sus características 


\section{Fibrilación auricular: factores de riesgo, diagnóstico y tratamiento}

Vol. 3, núm. 3 Esp., (2019)

Miguel Ángel Pozo Arcentales; María José Cedeño Zambrano; Karol Natalie Galarza Avila; Luis Javier Alvarez Moyon

electrocardiográficas diagnosticas son: la ausencia de una onda p sinusal, línea de base irregular, presencia de un intervalo R-R variable, frecuencia cardiaca auricular variable entre 350 a 600 latidos por minuto (lpm) y complejos QRS estrechos (< 120 milisegundos), en ausencia de otras alteraciones de la conducción; pudiendo presentarse como taquicardia de complejos QRS anchos (> 120 milisegundos) en pacientes con bloqueos de rama o conducción infrahisiana aberrante. Clínicamente puede ser asintomática o cursar con síntomas inespecíficos como disnea de esfuerzos, palpitaciones, angina o sincope. La mayoría de veces el diagnostico se hace mediante realización de electrocardiograma en el contexto de sus complicaciones (ECV, embolismo sistémico, IAM o falla cardiaca) $(1,2,4,5,7-9,14)$. El uso de dispositivos de monitoreo cardíaco (holter, grabadoras de bucle implantables, aplicaciones móviles, etc.) ha llevado a un aumento de la prevalencia en la detección de episodios de fibrilación auricular, por lo cual la duración de los episodios debe ser mayor a 30 segundos para hacer el diagnóstico.

\section{Metodología.}

Para el desarrollo de este proceso investigativo, se plantea como metodología la encaminada hacia una orientación científica particular que se encuentra determinada por la necesidad de indagar en forma precisa y coherente una situación, en tal sentido (Davila, 2015) define la metodología "como aquellos pasos previos que son seleccionados por el investigador para lograr resultados favorables que le ayuden a plantear nuevas ideas".(p.66).

Lo citado por el autor, lleva a entender que el desarrollo de la acción investigativa busca simplemente coordinar acciones enmarcadas en una revisión bibliográfica con el fin 


\section{Fibrilación auricular: factores de riesgo, diagnóstico y tratamiento}

Vol. 3, núm. 3 Esp., (2019)

Miguel Ángel Pozo Arcentales; María José Cedeño Zambrano; Karol Natalie Galarza Avila; Luis Javier Alvarez Moyon

de complementar ideas previas relacionadas a los factores de riesgo, diagnóstico y tratamiento de la fibrilación articular a través de una revisión de literatura, para así finalmente elaborar un cuerpo de consideraciones generales que ayuden a ampliar el interés propuesto.

Tipo de Investigación

Dentro de toda práctica investigativa, se precisan acciones de carácter metodológico mediante las cuales, se logra conocer y proyectar los eventos posibles que la determinan, así como las características que hacen del acto científico un proceso interactivo ajustado a una realidad posible de ser interpretada. En este sentido, se puede decir, que la presente investigación corresponde al tipo documental, definido por Castro (2016), "se ocupa del estudio de problemas planteados a nivel teórico, la información requerida para abordarlos se encuentra básicamente en materiales impresos, audiovisuales y /o electrónicos”. (p.41).

En consideración a esta definición, la orientación metodológica permitió la oportunidad de cumplir con una serie de actividades inherentes a la revisión y lectura de diversos documentos donde se encontraron ideas explicitas relacionadas con los tópicos encargados de identificar a cada característica insertada en el estudio. Por lo tanto, se realizaron continuas interpretaciones con el claro propósito de revisar aquellas apreciaciones o investigaciones propuestas por diferentes investigadores relacionadas con el tema de interés, para luego dar la respectiva argumentación a los planteamientos, en función a las necesidades encontradas en la indagación. 


\section{Fibrilación auricular: factores de riesgo, diagnóstico y tratamiento}

Vol. 3, núm. 3 Esp., (2019)

Miguel Ángel Pozo Arcentales; María José Cedeño Zambrano; Karol Natalie Galarza Avila; Luis Javier Alvarez Moyon

\section{Fuentes Documentales}

El análisis correspondiente a las características que predomina en el tema seleccionado, llevan a incluir diferentes fuentes documentales encargadas de darle el respectivo apoyo y en ese sentido cumplir con la valoración de los hechos a fin de generar nuevos criterios que sirven de referencia a otros procesos investigativos. Para (CASTRO, 2016) las fuentes documentales incorporadas en la investigación documental o bibliográfica, "representa la suma de materiales sistemáticos que son revisados en forma rigurosa y profunda para llegar a un análisis del fenómeno".(p.41). Por lo tanto, se procedió a cumplir con la realización de una lectura previa determinada para encontrar aquellos aspectos estrechamente vinculados con el tema, con el fin de explicar mediante un desarrollo las respectivas apreciaciones generales de importancia.

\section{Técnicas para la Recolección de la Información}

La conducción de la investigación para ser realizada en función a las particularidades que determinan a los estudios documentales, tiene como fin el desarrollo de un conjunto de acciones encargadas de llevar a la selección de técnicas estrechamente vinculadas con las características del estudio. En tal sentido, (Bolivar, 2015), refiere, que es "una técnica particular para aportar ayuda a los procedimientos de selección de las ideas primarias y secundarias”. (p. 71).

Por ello, se procedió a la utilización del subrayado, resúmenes, fichaje, como parte básica para la revisión y selección de los documentos que presentan el contenido teórico. Es decir, que mediante la aplicación de estas técnicas se pudo llegar a recoger informaciones en cuanto a la revisión bibliográfica de los diversos elementos encargados de orientar el proceso de investigación. Tal como lo expresa, (Bolivar, 2015) "las técnicas documentales proporcionan las 


\section{Fibrilación auricular: factores de riesgo, diagnóstico y tratamiento}

Vol. 3, núm. 3 Esp., (2019)

Miguel Ángel Pozo Arcentales; María José Cedeño Zambrano; Karol Natalie Galarza Avila; Luis Javier Alvarez Moyon

herramientas esenciales y determinantes para responder a los objetivos formulados y llegar a resultados efectivos" (p. 58). Es decir, para responder con eficiencia a las necesidades investigativas, se introdujeron como técnica de recolección el método inductivo, que hizo posible llevar a cabo una valoración de los hechos de forma particular para llegar a la explicación desde una visión general.

Asimismo, se emplearon las técnicas de análisis de información para la realización de la investigación que fue ejecutada bajo la dinámica de aplicar diversos elementos encargados de determinar el camino a recorrer por el estudio, según, (Bolívar, 2015) las técnicas de procesamiento de datos en los estudios documentales "son las encargadas de ofrecer al investigador la visión o pasos que debe cumplir durante su ejercicio, cada una de ellas debe estar en correspondencia con el nivel a emplear" (p. 123). Esto indica, que para llevar a cabo el procesamiento de los datos obtenidos una vez aplicado las técnicas seleccionadas, tales como: fichas de resumen, textual, registros descriptivos entre otros, los mismos se deben ajustar al nivel que ha sido seleccionado.

\section{Resultados.}

\section{Epidemiología}

Se considera la arritmia más frecuente en la práctica clínica. Su prevalencia global es $0.4 \%$, aumenta del $2 \%$ a $5 \%$ en pacientes mayores de 60 años y llega a su tope del $10 \%$ en mayores de 80 años (DePalma, 2016). Estudios recientes evidencian un aumento en su incidencia, siendo de 0,35 por 1.000 pacientes atendidos en 2010 , afectando principalmente a personas de sexo masculino (relación hombre: mujer de 1:1,27) de la séptima década de la vida y 


\section{Fibrilación auricular: factores de riesgo, diagnóstico y tratamiento}

Vol. 3, núm. 3 Esp., (2019)

Miguel Ángel Pozo Arcentales; María José Cedeño Zambrano; Karol Natalie Galarza Avila; Luis Javier Alvarez Moyon

con una tasa de mortalidad de 0,0468 por 1000 habitantes en el periodo 2000-2009 (Romero \& Chávez, 2014).

Es un factor de riesgo independiente para ECV con un aumento de 3 a 5 veces el riesgo de sufrirlo en pacientes no anticoagulados, siendo responsable de 1 de cada 6 eventos cerebrovasculares y del 20-50\% de los ECV cardioembólicos, con un incremento de mortalidad de 2 veces. $25 \%$ de los pacientes tienen formas asintomáticas que persisten por años. A pesar de saber que estos pacientes tienen aumento del riesgo de sufrir eventos tromboembólicos, su conocida asociación con mortalidad y la clara asociación de una reducción de este riesgo con el uso de anticoagulantes, la cantidad de pacientes anticoagulados en la actualidad es mucho menor de lo esperado. (Pinto, y otros, 2016) demostraron como entre los pacientes con FA que consultaron a urgencias en un centro de referencia de Bogotá, solo el 54\% de los pacientes con indicaciones estaban anticoagulados. Una de las principales razones para la baja tasa de prescripción de anticoagulantes sigue siendo el temor a los eventos de sangrado, a pesar de contar con opciones más seguras como los anticoagulantes orales directos que han demostrado tanto en estudios clínicos controlados como en escenarios de la vida real una menor tasa de sangrado cuando se comparan con warfarina (22.2\% para sangrado intracraneal y $31.2 \%$ para sangrado gastrointestinal) (Bouget \& Oger, 2015). La warfarina sigue siendo el anticoagulante oral más frecuentemente formulado a estos pacientes por su bajo costo. (Pinto, y otros, 2016) reportaron recientemente que solo el $2-3 \%$ de los pacientes tienen formas paroxísticas, con un riesgo de recurrencias de $10 \%$ el primer año y del $5 \%$ anual después del segundo año, evolucionando en su mayoría hacia formas permanentes. Tener tanto formas paroxísticas como persistentes/ permanentes genera el mismo riesgo de cardio embolismo 


\section{Fibrilación auricular: factores de riesgo, diagnóstico y tratamiento}

Vol. 3, núm. 3 Esp., (2019)

Miguel Ángel Pozo Arcentales; María José Cedeño Zambrano; Karol Natalie Galarza Avila; Luis Javier Alvarez Moyon

\section{Clasificación}

Actualmente existen dos clasificaciones recomendadas por la guía de práctica clínica de la Sociedad Europea de Cardiología de que la definen según su patrón y tipo clínico. Según el patrón o duración temporal, se puede clasificar en:

Primer episodio: episodio inicial en paciente sin diagnóstico previo, independientemente de la duración del evento y de los síntomas relacionados.

Paroxística: episodio que dura entre 48 horas y 7 días, incluidos aquellos casos que son cardiovertidos eléctrica o farmacológicamente en las primeras 48 horas.

Persistente: episodio que dura más de 7 días y menos de 1 año, incluidos los que con cardiovertidos farmacológica o eléctricamente después de 48 horas.

Persistente de larga duración: duración mayor o igual a 1 año en paciente que se ha decidido recuperar el ritmo sinusal.

Permanente: episodio que dura más de 1 año o aquel de duración inferior, en el cual, médico y paciente acuerdan que no se buscará recuperar el ritmo sinusal.

Según la clasificación de patrones de fibrilación auricular, la guía europea considera paroxística aquella que es cardiovertida en los primeros 7 días y persistente cuando se cardiovierte luego de este periodo de tiempo. 


\section{Fibrilación auricular: factores de riesgo, diagnóstico y tratamiento}

Vol. 3, núm. 3 Esp., (2019)

Miguel Ángel Pozo Arcentales; María José Cedeño Zambrano; Karol Natalie Galarza Avila; Luis Javier Alvarez Moyon

Según su tipo clínico o etiología:

Fibrilación auricular de origen valvular: Si bien las definiciones existentes en la literatura son múltiples; las guías de práctica clínica y dos revisiones recientes la definen como aquella que se presenta en pacientes con estenosis valvular mitral significativa (moderada a severa) o la presencia de prótesis valvulares mecánicas en cualquier posición. Esta definición permisiva pretende disminuir la cantidad de pacientes que son descartados del uso de anticoagulantes orales directos por el uso de definiciones más estrictas (Martins, y otros, 2016). Es importante aclarar que los estudios regulatorios de rivaroxaban y dabigatran excluyeron a pacientes con presencia de válvulas biológicas o enfermedad valvular significativa en cualquier posición con necesidad de cirugía durante el curso del estudio; por lo cual se recomienda que caso de decidir usar un anticoagulante oral directo en estos pacientes se prefiera el uso de apixaban o edoxaban, dado que en sus estudios se permitió la participación de pacientes con una definición permisiva.

Fibrilación auricular secundaria a cardiopatía estructural: aquella que se presenta en pacientes con disfunción ventricular izquierda sistólica o diastólica, HTA de larga duración asociada a hipertrofia ventricular y/o en pacientes con cualquier otra enfermedad cardiaca estructural.

Fibrilación auricular focal: se define como aquella caracterizada por carreras de taquicardia atrial asociadas a episodios cortos y frecuentes de FA paroxística, observándose ondas auriculares en el electrocardiograma (FA gruesa), ectopias auriculares y/o taquicardia auricular que degenera en FA. 


\section{Fibrilación auricular: factores de riesgo, diagnóstico y tratamiento}

Vol. 3, núm. 3 Esp., (2019)

Miguel Ángel Pozo Arcentales; María José Cedeño Zambrano; Karol Natalie Galarza Avila; Luis Javier Alvarez Moyon

Fibrilación auricular postoperatoria: aparición de FA en pacientes con ritmo sinusal conocido sin episo-dios de FA previos, que surge durante el periodo postquirúrgico de cirugía cardiaca mayor, usualmente autolimitada.

Fibrilación auricular del atleta: episodios de fibrilación auricular paroxística relacionada con la intensidad y duración del entrenamiento físico.

\section{Evaluación inicial}

Al igual que en cualquier patología, la anamnesis debe incluir tiempo de evolución de síntomas y establecer si este es el primer episodio o se trata de una recurrencia, orientándose hacia la búsqueda de enfermedades concomitantes, factores de riesgo y descartar causas reversibles; ya que de ello dependerá la terapéutica a largo plazo. Los principales hallazgos al examen físico que deben hacerla sospechar son déficit de pulso, frecuencia cardiaca variable y ruidos cardiacos irregulares. Los paraclínicos iniciales deben incluir hemograma, electrocardiograma, radiografía de tórax, reactantes de fase aguda, electrolitos, glucemia, enzimas cardiacas, perfil tiroideo, tiempos de coagulación, gases arteriales, función renal, uroanálisis y ecocardiograma transtóracico; los cuales están orientados a detectar la presencia de causas reversibles de la arritmia, enfermedad cardiaca estructural y la presencia de factores de riesgo cardiovascular. La realización de otras ayudas diagnosticas solo se indica según los resultados de los exámenes iniciales y según las condiciones clínicas y evolución del paciente, por lo cual no se recomiendan de rutina (Andrade, y otros, 2016).

Siempre deberá valorarse la calidad de vida de la paciente asociada con los síntomas por FA mediante el puntaje de EHRA, lo cual permitirá definir el tipo de terapia a ofrecer. La 


\section{Fibrilación auricular: factores de riesgo, diagnóstico y tratamiento}

Vol. 3, núm. 3 Esp., (2019)

Miguel Ángel Pozo Arcentales; María José Cedeño Zambrano; Karol Natalie Galarza Avila; Luis Javier Alvarez Moyon

evaluación integral e intervención de los factores de riesgo cardiovascular debe ser parte de toda atención a pacientes con FA, dado que está demostrado en estudios clínicos como su abordaje y manejo integral reduce morbilidad, mortalidad y disminuye la tasa de recurrencia de eventos de FA en pacientes con recuperación del ritmo sinusal y que sufren de hipertensión arterial y falla cardiaca. De igual forma deberá estarse atento a la presencia de signos de alarma en el paciente que indiquen la necesidad de hospitalización, referencia a una unidad especializada en fibrilación auricular o a especialistas en electrofisiología, como son inestabilidad hemodinámica, respuesta ventricular no controlable, bradicardia sintomática que no mejora con la reducción de dosis o interrupción de los antiarritmicos, angina severa, empeoramiento de la función ventricular, ataque isquémico transitorio o accidente cerebrovascular isquémico (DePalma, 2016).

\section{Control de ritmo}

El manejo antiarrítmico se divide en dos estrategias: control de respuesta ventricular y control de ritmo. La restauración del ritmo sinusal o cardioversión puede ser obtenida mediante cardio-versión eléctrica, cardioversión farmacológica, ablación percutánea o quirúrgica. Cualquiera de estos métodos es igualmente efectivo y la elección de uno sobre otro depende más de las condiciones del paciente o falla previa de uno de ellos; siendo la cardioversión eléctrica de elección en el paciente hemodinámicamente inestable, en quien la restauración del ritmo debe ser inmediata o en pacientes con contraindicaciones para las otras terapias (1, 5, 6, 8-10, 54-59). Las demás modalidades son ideales en pacientes estables hemodinámicamente, jóvenes ( $\leq 65$ años), con evolución corta de su arritmia ( $\leq 1$ año e idealmente $<48$ horas), sin cardiopatía estructural (aurícula izquierda $<50 \mathrm{~mm}$ y sin disfunción ventricular), con formas recurrentes y quienes persisten sintomáticos a pesar de estar en manejo óptimo de control de respuesta ventricular o 


\section{Fibrilación auricular: factores de riesgo, diagnóstico y tratamiento}

Vol. 3, núm. 3 Esp., (2019)

Miguel Ángel Pozo Arcentales; María José Cedeño Zambrano; Karol Natalie Galarza Avila; Luis Javier Alvarez Moyon

con refractariedad a otras terapias; teniendo en cuenta que ninguna de las opciones ha demostrado hasta ahora ser superior sobre la otra en términos de mortalidad, prevención de tromboembolismo o evolución a falla cardiaca. En pacientes con falla cardiaca, los estudios realizados no han logrado demostrar beneficios de mortalidad o desenlaces clínicos fuertes, excepto para pacientes con disfunción ventricular se-vera $(\mathrm{FEVI} \leq 35 \%)$, que permanecen en falla cardiaca sintomática (NYHA 3) a pesar de manejo optimo por 1 mes.

Un estudio comparando control de respuesta ventricular estricto contra control de ritmo por ablación con catéter en este grupo de pacientes, demostró mejoría de calidad de vida y niveles de BNP; lo cual está respaldado por un metaanálisis reciente de esta estrategia de manejo en pacientes con falla cardiaca, con una tasa de éxito dependiente del tiempo de evolución de la falla cardíaca y el método de ablación utilizado (Anselmino, y otros, 2014). Las características principales de los diferentes métodos de cardioversión y los factores que influencian su elección se escapan del alcance de este artículo y se remite al lector a sus estudios fundamentales (60-65), revisiones y a las guías de manejo de FA para obtener dicha información. Pese a sus efectos adversos, la amiodarona sigue siendo uno de los medicamentos más seguros y eficaces en lograr control del ritmo, con una relación costo-beneficio razonable cuando se usa adecuadamente. De otro lado, la digoxina solo se recomienda como terapia de segunda línea de control de frecuencia en pacientes con contraindicaciones para el uso de beta bloqueadores y calcioantagonistas, o en quienes su uso como monoterapia no ha logrado el control de la respuesta ventricular, dado su asociación con mayor mortalidad en los estudios retrospectivos que no ha podido ser confirmada en estudios prospectivos y su baja eficacia en control de ritmo. 


\section{Fibrilación auricular: factores de riesgo, diagnóstico y tratamiento}

Vol. 3, núm. 3 Esp., (2019)

Miguel Ángel Pozo Arcentales; María José Cedeño Zambrano; Karol Natalie Galarza Avila; Luis

Javier Alvarez Moyon

Figura 1. Resumen de manejo de fibrilación auricular

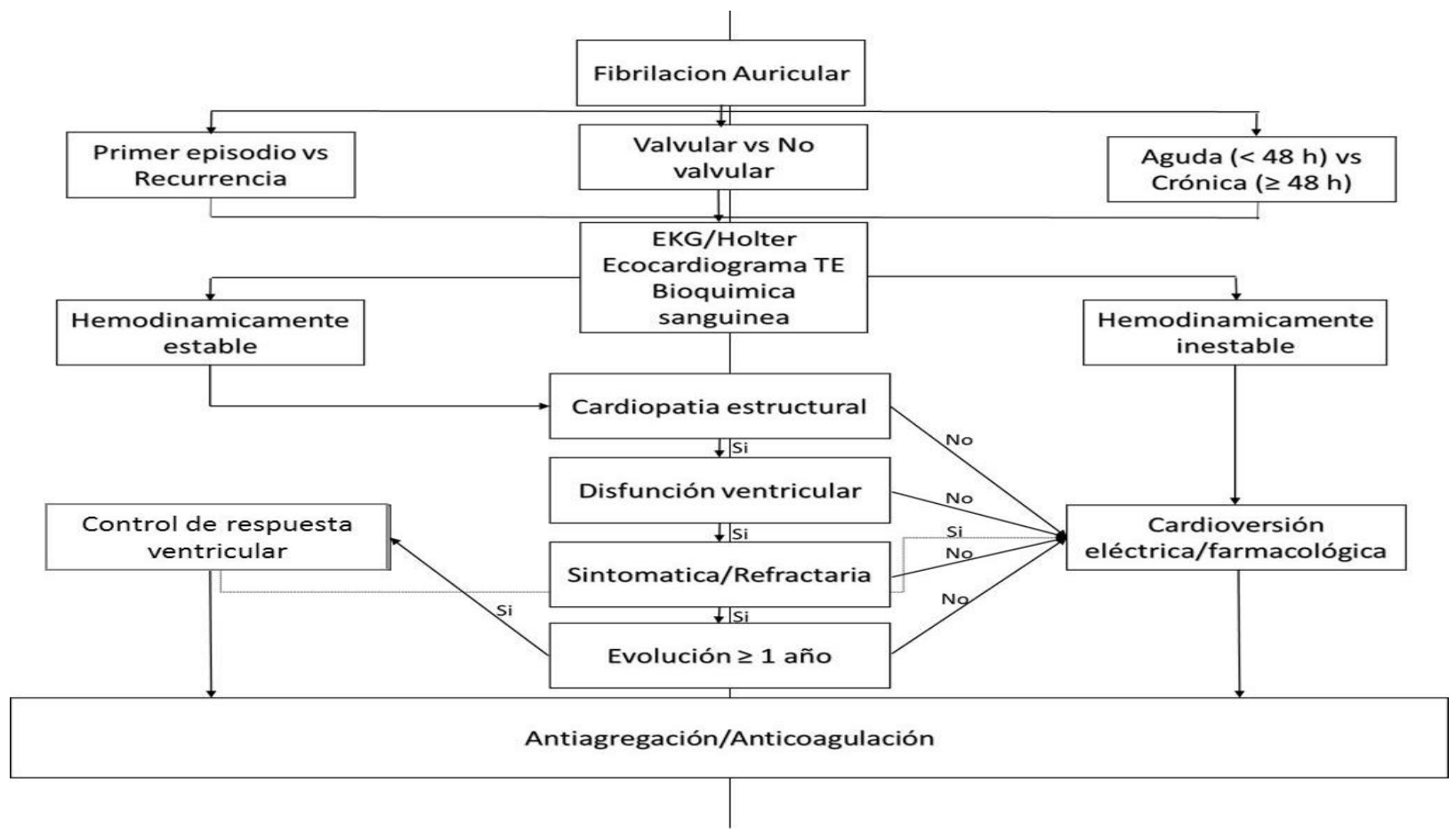

Control de respuesta ventricular

Se considera una alternativa terapéutica para pacientes con FA paroxística o persistente con síntomas leves, de edad avanzada (> 65 años), duración desconocida o prolongada (> 1 año) de la arritmia, múltiples comorbilidades y cardiopatía estructural de base (disfunción ventricular o dilatación auricular izquierda. Sus objetivos son controlar los síntomas del paciente, mejorar la calidad de vida, disminuir los efectos hemodinámicos y de remodelación para prevenir la taquicardiomiopatía. Antes de iniciarla se requiere descartar e identificar causas secundarias, realizar monitoreo Holter de 24 horas y prueba de esfuerzo en pacientes con síntomas asociados a la actividad física o que tengan factores de riesgo y/o síntomas de enfermedad coronaria. La frecuencia cardiaca (FC) objetivo inicial debe ser menor a $110 \mathrm{lpm}$ en reposo (control de 


\section{Fibrilación auricular: factores de riesgo, diagnóstico y tratamiento}

Vol. 3, núm. 3 Esp., (2019)

Miguel Ángel Pozo Arcentales; María José Cedeño Zambrano; Karol Natalie Galarza Avila; Luis Javier Alvarez Moyon

frecuencia permisivo). A la luz de la evidencia actual el buscar control de frecuencia estricto (FC menor a $80 \mathrm{lpm}$ en reposo y menor a $110 \mathrm{lpm}$ en ejercicio) solo se justifica cuando persisten síntomas asociados a taquiarritmia a pesar de control permisivo y compensación de comorbilidades. Lograr controlar la res-puesta ventricular es un reto difícil que a veces requeri-rá combinaciones de medicamentos y aumento de dosis de los mismos según el puntaje EHRA y registros de monitoreo Holter; teniendo siempre en cuenta su potencial proarrítmico.

\section{Recomendaciones para la anticoagulación}

La recomendación de anticoagulación es universal para todo paciente con al menos un episodio de FA documentado y en quien no exista una causa corregible de la misma; recomendándose incluso en este último caso el uso de anticoagulación a corto plazo y la posterior consideración de interrupción de la misma tras haberse documentado ausencia de nuevos eventos durante un periodo de tiempo prudente que puede oscilar entre 3 a 6 meses. Para pacientes que han sido sometidos a terapias "curativas" de FA (cardioversión eléctrica o farmacológica, ablación auricular, aislamiento de venas pulmonares, cirugía de Maze, cierre de apéndice auricular, etc.) se recomienda mantener la anticoagulación en forma indefinida siempre que no existan contraindicaciones claras para esta, dada la alta probabilidad de eventos de FA recurrentes asintomáticos que pueden llevar a eventos cardioembólicos si se retira la anticoagulación (Aristizábal, y otros, 2012).

\section{Conclusiones.}

La FA es una enfermedad prevalente en nuestro medio, que cada vez cobra una importancia mayor, a medida que la población mejora su expectativa de vida y envejece. Esta es 


\section{Fibrilación auricular: factores de riesgo, diagnóstico y tratamiento}

Vol. 3, núm. 3 Esp., (2019)

Miguel Ángel Pozo Arcentales; María José Cedeño Zambrano; Karol Natalie Galarza Avila; Luis Javier Alvarez Moyon

una enfermedad asociada a mayor morbilidad y mortalidad cardiovascular potencialmente discapacitante. Si bien se ha avanzado y aumentado la posibilidad de diagnóstico, es importante que todo el personal médico esté capacitado para realizar su manejo adecuado; evitándose así las complicaciones con sus implicaciones personales, sociales y económicas, teniendo en cuenta el control de ritmo/respuesta ventricular y la anticoagulación oral como los principales pilares en su manejo. El control de respuesta ventricular está dirigido a controlar lo síntomas, debiendo considerarse el control de ritmo en pacientes con falla cardiaca asociada o síntomas persistentes a pesar de control de respuesta ventricular permisiva. Ninguna de estas estrategias ha demostrado disminuir la mortalidad atribuible a la enfermedad. La anticoagulación oral está indicada en los pacientes con FA y alto riesgo de presentar un fenómeno cardioembólico, debiendo individualizarse la decisión en pacientes con puntuaciones menores, con riesgo alto de sangrado o en quienes presenten otras indicaciones de anticoagulación diferentes a las incluidas en los puntajes de riesgo. Esta terapia ha demostrado disminuir la morbilidad y mortalidad secundarias a FA por lo cual solo está justificado no usarla en aquellos pacientes con verdaderas contraindicaciones para su uso que no sean controlables o corregibles; pudiendo en estos pacientes considerar otras alternativas al manejo farmacológico antitrombótico como el cierre/oclusión de orejuela de la aurícula izquierda para disminuir el riesgo de tromboembolismo. La warfarina sigue siendo el estándar de oro de anticoagulación por lo cual se debe buscar optimizar su uso, la cual estará contraindicada en aquellos pacientes con alta probabilidad de fallo a la terapia, que no puedan acceder a un seguimiento estrecho por clínica de anticoagulación o en quienes el riesgo de sangrado sea alto. En estos pacientes el uso de anticoagulantes orales directos estaría justificado bajo una perspectiva de costo - efectividad, teniendo en cuenta que dabigatran y apixaban son 


\section{Fibrilación auricular: factores de riesgo, diagnóstico y tratamiento}

Vol. 3, núm. 3 Esp., (2019)

Miguel Ángel Pozo Arcentales; María José Cedeño Zambrano; Karol Natalie Galarza Avila; Luis Javier Alvarez Moyon

las mejores opciones. En caso de presentarse sangrado con el uso de anticoagulantes, la interrupción de la terapia deberá ser solo transitoria, debiéndose reiniciar en el menor tiempo posible, una vez se controle la fuente del sangrado y corrijan los factores de riesgo adicionales para sangrado recurrente.

\section{Bibliografía.}

Andrade, J. G., Verma , A., Mitchell, L. B., Parkash , R., Leblanc, K., Atzema, C., . . Macle , L. (2016). Focused Update of the Canadian Cardiovascular Society Guidelines for the Management of Atrial Fibrillation. Can J Cardiol, 34(11), 1371-1392.

Anselmino, M., Matta, M., D’Ascenzo, F., Bunch, T., Schilling, R., \& Hunter, R. (2014). Catheter ablation of atrial fibrillation in patients with left ventricular systolic dysfunction: a systematic review and meta-analysis. Circ Arrhythm Electrophysiol, 7(6), 1011-8.

Aristizábal, J., Uribe, W., Medina, E., Velásquez, J., Ma-rín, J., \& Duque, M. (2012). Fibrilación auricular: una mirada actual. Rev Colomb Cardiol, 19(5), 235-251.

Bolívar, J. (2015). Investigación Documental. México: Pax.

Bouget, J., \& Oger, E. (2015). Emergency admissions for major haemorrhage associated with direct oral anticoa-gulants. Thromb Res, 136(6), 1190-4.

Castro, J. (2016). Técnicas Documentales. México: Limusa.

Davila, A. (2015). Concepto de terminos cientificos. Caracas: Oasis.

DePalma, S. (2016). Managing atrial fibrillation in primary care. JAAPA, 29(6), 29-33.

Martins, R., Galand, V., Colette, E., Behar, N., Pavin, D., \& Leclercq, C. (2016). Defining nonvalvular atrial fibri-llation: A quest for clarification. Am Heart J., 178, 161-167.

Pava-Molano, L., \& Perafán-Bautista, P. (2016). Generalidades de la fibrilación auricular. Rev Colomb Cardiol, 23(5), 5-8.

Pinto, D., Sánchez-Vallejo, C., López, A., Vergara, E., Sáenz, O., \& González, F. (2016). Descripción de los pacientes con fibrilación auricular no valvular que ingresan al servicio de urgencias. Rev Colomb Cardiol, 23(4), 270-276.

Romero, M., \& Chávez, D. (2014). Carga de enfermedad atribui-ble a fibrilación auricular en Colombia (2000-2009). Rev Colomb Cardiol, 21(6), 374-381. 
Fibrilación auricular: factores de riesgo, diagnóstico y tratamiento

Vol. 3, núm. 3 Esp., (2019)

Miguel Ángel Pozo Arcentales; María José Cedeño Zambrano; Karol Natalie Galarza Avila; Luis Javier Alvarez Moyon

Roselli, D., Rodríguez, A., García, A., \& Rueda, J. (2013). Prevalencia de fibrilación auricular en un hospital universitario colombiano. Rev Colomb Cardiol, 20(6), 383-385.

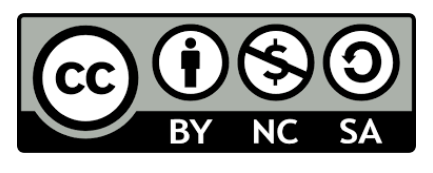

RECONOCIMIENTO-NOCOMERCIAL-COMPARTIRIGUAL

CC BY-NC-SA

ESTA LICENCIA PERMITE A OTROS ENTREMEZCLAR, AJUSTAR Y CONSTRUIR A PARTIR DE SU OBRA CON FINES NO

COMERCIALES, SIEMPRE Y CUANDO LE RECONOZCAN LA AUTORÍA Y SUS NUEVAS CREACIONES ESTÉN BAJO UNA LICENCIA CON LOS MISMOS TÉRMINOS. 